\title{
Serum S100A8 as an early diagnostic biomarker in patients with community-acquired pneumonia
}

Pu Fang ${ }^{1}$, Ling Zheng ${ }^{1}$, Peng $\mathrm{CaO}^{1}$, Chen Zhang ${ }^{1}$, Jun Fei ${ }^{1}$, Zheng Xu ${ }^{1}$, Chun-Mei Feng ${ }^{1}$, Hui Zhao ${ }^{1}$, You-Jin $\mathrm{Lu}^{1}$, Lin $\mathrm{Fu}^{1,2}$

\author{
${ }^{1}$ Respiratory and Critical Care Medicine, Second Affiliated Hospital of Anhui Medical \\ University, Hefei, China \\ ${ }^{2}$ Department of Toxicology of Anhui Medical University, Hefei, China
}

Submitted: 7 September 2020

Accepted: 19 November 2020

Arch Med Sci

DOI: https://doi.org/10.5114/aoms/130648

Copyright $\odot 2020$ Termedia \& Banach

\section{Abstract}

Introduction: Limited studies have suggested that calprotectin may take part in the pathophysiology of community-acquired pneumonia (CAP). Nevertheless, there is no clinical study analysing the role of S100A8 in CAP patients. The objective of this study was to analyse the association of serum S100A8 with the severity of CAP and determine the cut-off values of S100A8 for predictive power based on a cross-sectional study.

Material and methods: A total of 200 CAP patients and 100 normal subjects were recruited. Demographic data, clinical information, and serum were collected on admission. S100A8 and inflammatory cytokines were detected using ELISA and RT-PCR. All statistical analyses were performed with SPSS 19.0.

Results: Serum S100A8 was increased in CAP patients on admission. Serum S100A8 was gradually increased in parallel with CAP severity scores. Serum S100A8 was positively correlated with CAP severity scores, blood routine parameters, and inflammatory cytokines. Furthermore, univariate and multivariate logistical regression revealed that there were positive associations between serum S100A8 with CRB-65, PSI, and CURXO. Moreover, the predictive capacity of serum S100A8 was determined by ROC curve analysis. The area under the curves of S100A8 for CAP and CAP severity were 0.855 and 0.893 , respectively. Mechanistic analysis found that S100A8 knockdown alleviated streptococcus pneumoniae-evoked inflammatory cytokines in A549 cells.

Conclusions: Serum S100A8 on admission was positively associated with the severity of CAP. S100A8 knockdown alleviates streptococcus pneumoniae-evoked inflammatory cytokines in A549 cells, indicating that S100A8 may exert a significant effect on the pathophysiology of CAP and could be an early serum diagnostic biomarker for CAP.

Key words: community-acquired pneumonia, S100A8, inflammatory cytokines, community-acquired pneumonia severity score, blood routine, receiver operating characteristic, biomarker.

\section{Introduction}

Community-acquired pneumonia (CAP) is an infectious disease caused by bacteria, viruses, or a combination of these infectious agents [1]. This disease is prevalent among people aged 50-60 years and under 5 years, and always emerges when it is cold, especially in winter and

\author{
Corresponding authors: \\ Hui Zhao \\ You-Jin Lu \\ Lin Fu \\ Respiratory and Critical \\ Care Medicine \\ the Second Affiliated \\ Hospital \\ Anhui Medical \\ University \\ 678 Furong Road \\ Hefei 230601 \\ China \\ Phone: +8655165997174 \\ E-mail: fulindev@126.com \\ zhaohuichenxi@126.com \\ luyougolden@hotmail.com
}


early spring [2]. Currently, CAP is increasingly common worldwide and responsible for significant morbidity and mortality [3]. Assessment of severity and the risk for pneumonia at the time of initial diagnosis are necessary for optimal pneumonia management, including selection of the best site of care (outpatient, inpatient general ward, or ICU) $[4,5]$. However, early severity assessment and risk stratification for CAP are challenging because obvious clinical characteristics at an early stage are not highly predictive of which patients will suffer from deterioration of their condition. The severity of the clinical manifestations in CAP patients varies significantly [6]. CAP has become a serious growing public health issue and medical curiosity, and it elevates emotional and financial pressure to family, society, and the government [7]. Therefore, it is beneficial to seek biomarkers for diagnosis, prediction of severity, and identification of ways to reduce the incidence or severity.

Calprotectin (S100A8 and S100A9) comprises $\mathrm{Ca}^{2+}$ binding proteins belonging to the $\mathrm{S} 100 \mathrm{fam}$ ily, which are expressed in a wide variety of cell types and are abundant in myeloid cells, such as neutrophils, monocytes, keratinocytes, and early differentiation states of macrophages [8, 9]. S100A8 and S100A9 form non-covalently associated complexes that exhibit typical properties of damage-associated molecular patterns (DAMPs), which are released by activated granulocytes and act in a cytokine-like manner through binding to cell surface receptors, such as toll-like receptor 4 (TLR4), scavenger receptor CD36, or receptor of advanced glycation end products (RAGE), which triggers signalling pathways involved in the inflammatory processes and plays critical roles in numerous cellular processes [10-13]. Several studies found that S100A8 was increased in inflammatory diseases, including inflammatory bowel disease, chronic obstructive pulmonary disease, rheumatoid arthritis, cystic fibrosis, autoimmune diseases, and neurodegenerative disorders [14-18]. It is suggested that S100A8 might be considered as a significant biomarker for diagnostic purposes.

An earlier animal experiment found high expression of S100A8 protein in the pulmonary alveolar walls in mice infected with streptococcus pneumoniae [19]. In vitro experimentation demonstrated that S100A8 protein was increased in the bronchial epithelium after inflammation stimulation [20]. In addition, S100A8 was highly abundant and secreted into lung lavage fluid in mice after lipopolysaccharide exposure [21]. These data indicated that S100A8 might play an important role in infectious diseases. However, the role of S100A8 protein in CAP is still unclear. Therefore, we speculate that S100A8 heterodimer may take part in the pathogenesis of CAP. Nevertheless, there is no clinical and experiment research demonstrating the role of S100A8 heterodimer in CAP. Hence, the main goal of this study was to explore the correlations between serum S100A8 heterodimer with the severity of CAP and inflammatory cytokines, and to determine the cut-off values of S100A8 for predictive power in CAP patients with a population-based retrospective cross-sectional study.

\section{Material and methods}

\section{Subjects}

The Second Affiliated Hospital of Anhui Medical University is a tertiary care university hospital in Hefei City, Anhui Province, China. This retrospective study was performed in the Department of Respiratory and Critical Care Medicine from May 2018 to May 2020. This study was approved by the Ethics Committee of the Second Affiliated Hospital of Anhui Medical University. In total, 200 CAP patients (112 males and 88 females) and 100 healthy subjects were recruited in this study. Demographic and clinical information were collected on admission. All CAP patients gave advanced written and oral agreement for their inclusion and signed a consent form for this study. For CAP patients, the inclusion criteria consisted of inpatients more than 30 years old, who were diagnosed and admitted for treatment of CAP in the intensive care units or general wards. Exclusion criteria included being an outpatient; with other pulmonary diseases, serious complications, tuberculosis, malignancy, and asthma; having an organ or bone marrow transplant; being severely immunocompromised. All CAP patients were given empirical antimicrobial agents intravenously within the first $48 \mathrm{~h}$. Thereafter, antibiotics were given either orally or intravenously based on established guidelines. Pneumonia severity was evaluated by the CAP severity score, including the Pneumonia Severity Index (PSI), CURB-65 score, CRB-65 score, CURXO score, and SMART-COP score [22]. Blood samples were collected on admission before treatment. One hundred healthy subjects were randomly enrolled from the physical examination centre in the Second Affiliated Hospital of Anhui Medical University. The following fundamental data were collected from the electronic medical records of CAP patients and healthy subjects: demographic information, pre-existing comorbidities, symptoms and signs of CAP, and laboratory examination data.

\section{Cell culture}

Human pulmonary epithelial (A549) cell line was obtained from the American Type Culture Collection (USA). A549 cells were cultured with RPMI 1640 medium (HyClone; Logan, UT) supplemented with $7.5 \%$ foetal bovine serum (FBS), $100 \mathrm{U} / \mathrm{ml}$ 
penicillin, and $100 \mathrm{mg} / \mathrm{ml}$ streptomycin in a humidified chamber at $37^{\circ} \mathrm{C}$ with $5 \% \mathrm{CO}_{2}$. Streptococcus pneumoniae was used for stimulations at a concentration of $10^{7} \mathrm{CFU} / \mathrm{ml}$ based on a previous study [23]. A549 cells were seeded into $25-\mathrm{cm}^{2}$ culture dishes. When the cells were cultured to $50 \%$ density, all cells were incubated with streptococcus pneumoniae for $12 \mathrm{~h}$. Cell supernatant were collected for enzyme-linked immunosorbent assay (ELISA). After $12 \mathrm{~h}$, the cells were washed with chilled PBS three times and then harvested for real-time RT-PCR.

\section{Small interfering RNA (siRNA) transfection}

Human S100A8 and scrambled siRNAs were obtained from the Gene Pharma Corp (Shanghai, China). The S100A8 siRNA sequences was as follows: (sense, 5'-GGUCACUACUGAGUGCCCUCAGUUU-3'; antisense, 5'- AAACUGAGGGCACUCAGUAGUGACC-3'). The RNA interference protocol was on the basis of a previous study [24]. Human S100A8-siRNA was transfected into cells using Lipofectamine 3000 according to the manufacturer's instructions (Invitrogen, Carlsbad, CA, United States). After 4-h incubation, the medium was replaced with fresh RPMI-1640 and the cells were incubated for another $48 \mathrm{~h}$. After siRNA transfection for $48 \mathrm{~h}$, streptococcus pneumoniae was continued to co-culture and A549 cells were harvested.

\section{Isolation of total RNA and real-time RT-PCR}

Total RNA from human lung epithelial cells were extracted using TRI reagent followed by a cleaning step $[25,26]$. The purified RNA was transcribed into cDNA using avian myeloid leukaemia virus reverse transcriptase (Promega) and analysed by real-time RT-PCR with a Light Cycler 480 SYBR Green I kit (Roche Diagnostics). The levels of mRNAs were calculated using the standard curve method and expressed as fold of control expression. Rn18S expression was used for the normalisation of each protein expression level for all samples.

\section{Enzyme-linked immunosorbent assay (ELISA)}

S100A8 ELISA kits were prepared from Wuhan ColorfulGene Biological Technology Co., Ltd. CRP, TNF- $\alpha$, IL- $1 \beta$, and IL- 6 ELISA kits were purchased from Cusabio, Wuhan, China (https://www.cusabio.com/). All cytokines were detected on the basis of the manufacturer's instructions [27].

\section{Statistical analysis}

All statistical analyses were performed using SPSS 19.0. Student's $t$ tests, $\chi^{2}$ tests, and Mann-Whitney $U$ tests were used to compare the demographic characteristics of means, propor- tions, and medians, respectively. Linear regression analyses were used to examine the associations between S100A8 and pneumonia severity scores, inflammatory cytokines, and blood routine parameters. Moreover, logistical regression analyses were performed between serum S100A8 and CAP severity scores. Categorical variables were expressed with frequencies and percentages. Continuous variables were shown using median and mean values. Statistical significance was determined at $p<0.05$.

\section{Results}

\section{Demographic and clinical information}

Altogether 200 CAP patients and 100 healthy subjects were rerolled in the Second Affiliated Hospital of Anhui Medical University. The demographic and clinical information was analysed between CAP patients and healthy subjects. As shown in Table I, no obvious difference in age, sex, body mass index (BMI), systolic pressure, and diastolic pressure was observed among the two groups. Moreover, the cases of comorbidities with hypertension, diabetes mellitus, interstitial pneumonia, chronic obstructive pulmonary disease, cor pulmonale, chronic kidney disease, and neurological disease were more common in CAP patients than in normal subjects. There was no difference in chronic liver disease, cardiovascular disease, and other diseases between two groups. Eighteen (9.0\%) CAP patients died after hospitalisation. The average hospital stay was 10.0 days. In addition, pneumonia severity was evaluated using CAP severity score. Of 200 CAP patients, 82 (41.0\%) severe patients were from the CAP cases (CURXO score), and the median PSI score, CURB-65 score, CRB-65 score, and SMART-COP score were 2.0, 1.0, 92.0 , and 2.0 , respectively.

\section{The levels of serum S100A8 heterodimer in control subjects and CAP patients}

Serum S100A8 heterodimer was measured in CAP patients and control subjects. As shown in Figure $1 \mathrm{~A}$, serum S100A8 was obviously increased in CAP patients compared with those in control subjects (69.23 pg/ml vs. $163.32 \mathrm{pg} / \mathrm{ml})$. Also, serum S100A8 heterodimer was analysed among different grades of CAP patients. As shown in Figure $1 \mathrm{~B}$, serum S100A8 heterodimer was increased in 0 score grade compared to those in 1-2 score grade and $\geq 3$ score grade based on CRB- 65 score. Serum S100A8 was higher in $\geq 3$ score grade than those in 1-2 score grade. According to CURB-65 score, serum S100A8 heterodimer was gradually increased in parallel with CURB-65 score (Figure 1 C). Additionally, we found that serum S100A8 heterodimer was higher in severe CAP patients than in mild CAP patients (CURXO score) (Figure $1 \mathrm{D}$ ). 
Pu Fang, Ling Zheng, Peng Cao, Chen Zhang, Jun Fei, Zheng Xu, Chun-Mei Feng, Hui Zhao, You-Jin Lu, Lin Fu

Table I. Demographic and biochemical characteristics of CAP patients and control subjects

\begin{tabular}{|c|c|c|c|}
\hline Variables & $\operatorname{CAP}(n=200)$ & Control $(n=100)$ & $P$-value \\
\hline Age [years] & $66.5(56.5,78.0)$ & $62.0(51.6,73.8)$ & 0.452 \\
\hline Male, $n(\%)$ & $112(56.0)$ & $108(54.0)$ & 0.321 \\
\hline BMI & $22.6(20.1,25.6)$ & $21.3(19.2,24.3)$ & 0.221 \\
\hline Systolic pressure [mm Hg] & $122.5(108.0,135.0)$ & $120.1(103.5,139.5)$ & 0.564 \\
\hline Diastolic pressure $[\mathrm{mm} \mathrm{Hg}]$ & $73.0(66.3,80.8)$ & $78.0(60.5,84.6)$ & 0.521 \\
\hline \multicolumn{4}{|l|}{ Comorbidities, $n$ (\%): } \\
\hline Hypertension & $44(22.0)$ & $9(9.0)$ & 0.005 \\
\hline Diabetes mellitus & $24(12.0)$ & 0 & $<0.001$ \\
\hline Interstitial pneumonia & $18(9.0)$ & 0 & 0.002 \\
\hline COPD & $12(6.0)$ & 0 & 0.012 \\
\hline Cor pulmonale & $16(8.0)$ & 0 & 0.004 \\
\hline Chronic liver disease & $20(10.0)$ & $4(4.0)$ & 0.071 \\
\hline Chronic heart disease & $6(3.0)$ & $3(3.0)$ & 1.000 \\
\hline Chronic kidney disease & $8(4.0)$ & 0 & 0.043 \\
\hline Cardiovascular disease & $21(10.5)$ & $5(5.0)$ & 0.110 \\
\hline Neurological disease & $10(5)$ & 0 & 0.023 \\
\hline Other disease & $31(15.5)$ & $12(12.0)$ & 0.415 \\
\hline Hospital stay [day] & $10.0(7.0,17.0)$ & N.A & N.A \\
\hline Mortality, $n(\%)$ & $18(9.0)$ & N.A & N.A \\
\hline CURB-65 & $2.0(0,3.0)$ & N.A & N.A \\
\hline CRB-65 & $1.0(0,2.0)$ & N.A & N.A \\
\hline PSI & $92.0(58.0,128.0)$ & N.A & N.A \\
\hline CURXO [Severe, $n(\%)]$ & $82(41.0)$ & N.A & N.A \\
\hline SMART-COP & $2.0(0,5.0)$ & N.A & N.A \\
\hline
\end{tabular}

Also, on the basis of SMART-COP score, serum S100A8 heterodimer in 0-2 score grade was lowest and in 7-8 score grade was highest, and serum S100A8 heterodimer in 5-6 score grade was higher than those in 0-2 score grade (Figure $1 \mathrm{E}$ ). In addition, serum S100A8 heterodimer was compared among different grades of CAP patients based on PSI score. As shown in Figure $1 \mathrm{~F}$, serum S100A8 heterodimer was gradually elevated in parallel with PSI score.

\section{Associations of S100A8 heterodimer with} disease severity, blood routine parameters, and inflammatory cytokines in CAP patients

The association between S100A8 heterodimer and disease severity was analysed among CAP patients. As shown in Table II, serum S100A8 heterodimer was positively and significantly associ- ated with CURB-65 $(r=0.541, p<0.001)$, CRB-65 $(r=0.598, p<0.001)$, PSI $(r=0.611, p<0.001)$, CURXO $(r=0.546, p<0.001)$, and SMART-COP $(r=0.492, p<0.001)$. Moreover, the associations between serum S100A8 heterodimer and blood routine parameters were evaluated. There was no obvious association between serum S100A8 with neutrophil and platelet-lymphocyte ratio (PLR). Serum S100A8 heterodimer was weakly and positively associated with white blood cell (WBC) $(r=0.294, p=0.003)$, NLR (neutrophil-lymphocyte ratio) ( $r=0.319, p<0.001)$, and monocyte-lymphocyte ratio (MON) $(r=0.223, p=0.027)$. Additionally, the associations of serum S100A8 and inflammatory cytokines were calculated. As shown in Table II, serum S100A8 was positively and significantly associated with TNF- $\alpha(r=0.396$, $p=0.005), \mathrm{IL}-1 \beta(r=0.310, p<0.001)$, and CRP $(r=0.345, p=0.027)$. There was no significant 
A

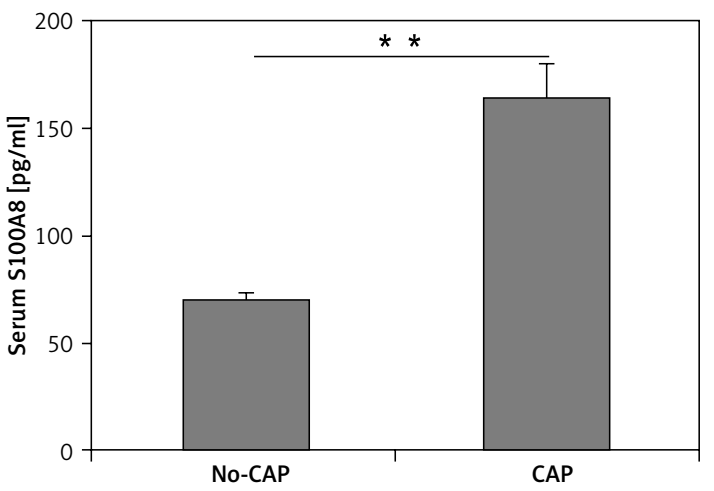

C

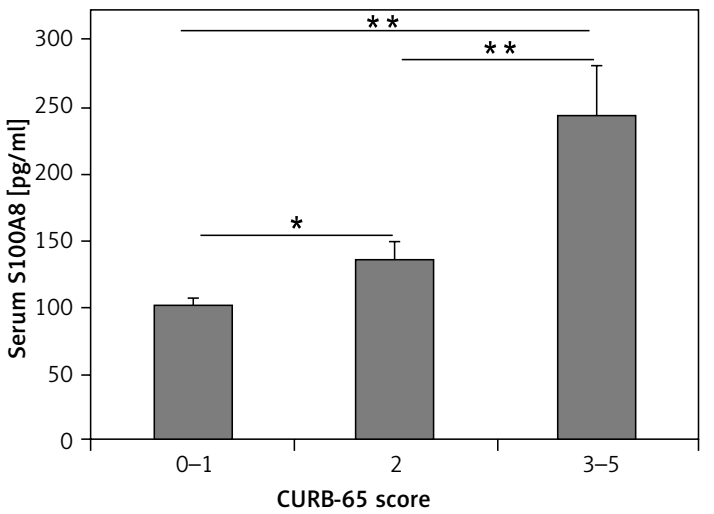

E

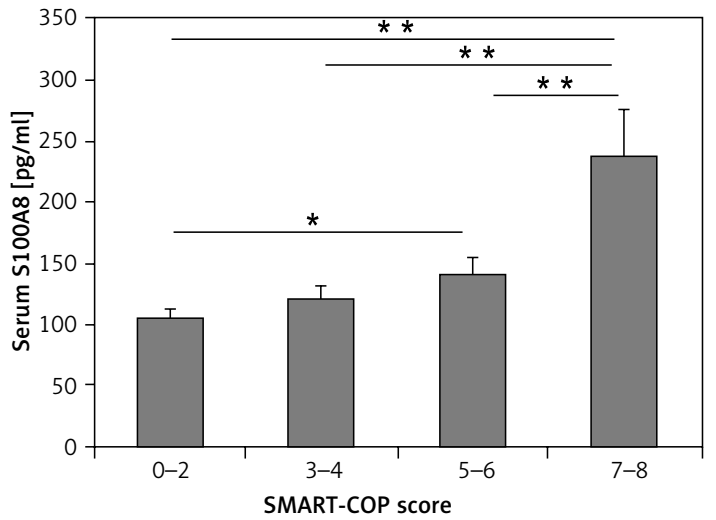

B

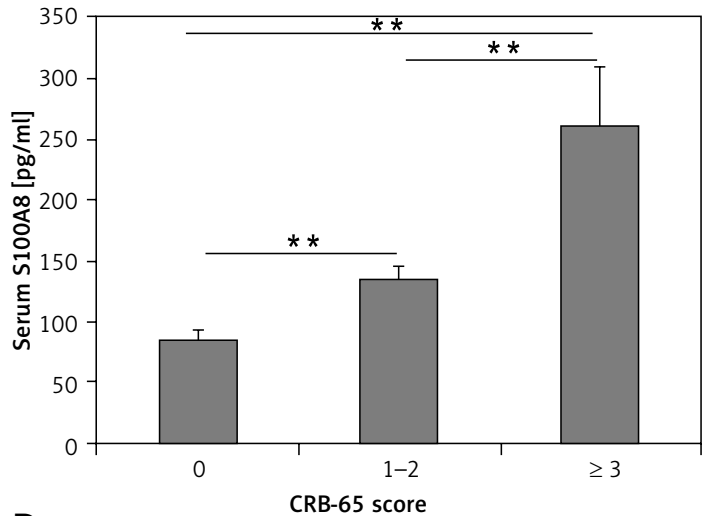

D

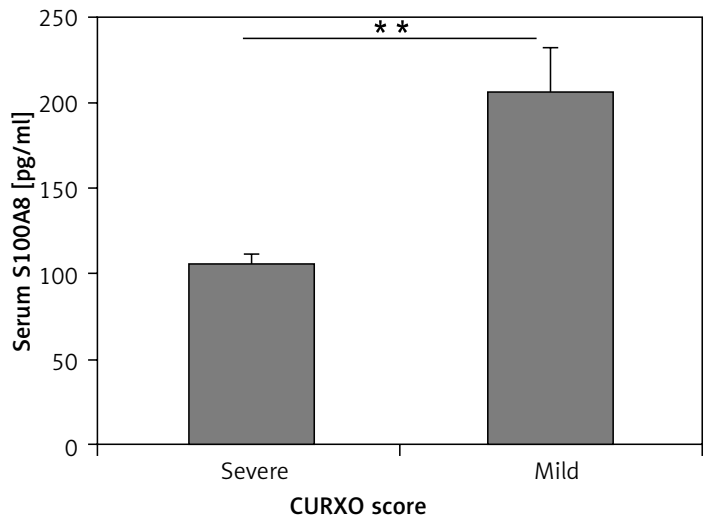

$\mathrm{F}$

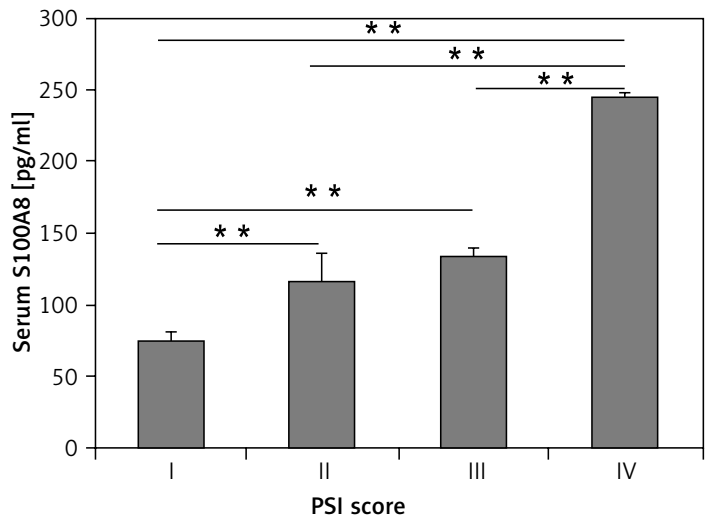

Figure 1. Serum S100A8 levels between CAP patients and control subjects. A - Serum S100A8 levels in CAP patients and normal subjects ( $n=100$ for normal subjects; $n=200$ for CAP patients). B-F - Serum S100A8 levels in different grades of CAP severity. B - CRB-65 score. C - CURB-65 score. D - CURXO score. E - SMART-COP sCore. $\mathrm{F}-\mathrm{PSI}$ score. All data were represented as mean \pm SEM. ${ }^{\star} P<0.05,{ }^{* *} p<0.01$

association between serum S100A8 and IL-6 ( $r=$ $0.398, p=0.055)$. Furthermore, associations of serum S100A8 with CAP severity indices were analysed with univariate logistic regression among CAP patients. As shown in Table III, serum S100A8 level was evidently and positively associated with CURB-65 ( $\beta=1.286$; 95\% Cl: 1.050-1.786), CRB-65 $(\beta=1.345 ; 95 \% \mathrm{Cl}: 1.004-2.415)$, PSI $(\beta=1.245 ; 95 \% \mathrm{Cl}: 1.009-1.894)$, SMART-COP $(\beta=1.286 ; 95 \% \mathrm{Cl}: 1.050-1.786)$, and CURXO $(\beta=1.018 ; 95 \% \mathrm{Cl}: 1.007-1.030)$. In order to con- trol confounders, associations of serum S100A8 with CAP severity score were furtherly analysed with multivariate logistic regression. However, there was no obvious association between serum S100A8 with CURB-65 and SMART-COP after adjustment for confounders (such as age, sex, BMI, and comorbidities). We found that serum S100A8 level was significantly and positively correlated with CRB-65 ( $\beta=1.112$; 95\% Cl: 1.001-1.421), PSI $(\beta=1.121 ; 95 \% \mathrm{Cl}: 1.004-1.415)$, and CURXO ( $\beta=1.118 ; 95 \% \mathrm{Cl}: 1.012-1.321$ ) (Table III). 
Table II. Correlations between serum S100A8 and other parameters

\begin{tabular}{|c|c|c|c|c|c|c|}
\hline \multirow[t]{3}{*}{ Severity score } & & CURB-65 & CRB-65 & PSI & CURXO & SMART-COP \\
\hline & $r$ & 0.541 & 0.598 & 0.611 & 0.546 & 0.492 \\
\hline & $P$-value & $<0.001$ & $<0.001$ & $<0.001$ & $<0.001$ & $<0.001$ \\
\hline \multirow[t]{3}{*}{ Blood routine } & & WBC & Neutrophil & NLR & MON & PLR \\
\hline & $r$ & 0.294 & 0.156 & 0.319 & 0.223 & -0.028 \\
\hline & $P$-value & 0.003 & 0.126 & $<0.001$ & 0.027 & 0.784 \\
\hline \multirow{3}{*}{$\begin{array}{l}\text { Inflammatory } \\
\text { cytokines }\end{array}$} & & TNF- $\alpha$ & IL-1 $\beta$ & CRP & IL-6 & \\
\hline & $r$ & 0.396 & 0.310 & 0.345 & 0.398 & \\
\hline & $P$-value & 0.005 & $<0.001$ & 0.002 & 0.055 & \\
\hline
\end{tabular}

Table III. Associations between serum S100A8 with CAP severity scores among CAP patients

\begin{tabular}{|llccc|}
\hline Variables & Univariable $(95 \% \mathrm{Cl})$ & $P$-value & Multivariable $(95 \% \mathrm{CI})^{*}$ & $P$-value \\
\hline CURB-65 & $1.286(1.050,1.786)$ & 0.003 & $1.007(0.993,1.022)$ & 0.312 \\
\hline CRB-65 & $1.345(1.004,2.415)$ & 0.001 & $1.112(1.001,1.421)$ & 0.040 \\
\hline PSI & $1.245(1.009,1.894)$ & 0.001 & $1.121(1.004,1.415)$ & 0.031 \\
\hline SMART-COP & $1.286(1.050,1.786)$ & 0.003 & $1.017(0.998,1.035)$ & 0.085 \\
\hline CURXO & $1.018(1.007,1.030)$ & 0.001 & $1.118(1.012,1.321)$ & 0.038 \\
\hline
\end{tabular}

Dependent variables: S100A8. Independent variable: CURB-65, CRB-65, PSI, SMART-COP, CURXO. *Adjusted for age, sex, BMI, and comorbidities.

\section{ROC curves and cut-off point analysis for serum S100A8}

Based on the data shown so far, an evaluation of the predictive capacity of serum S100A8 was performed by receiver operating characteristic area under the curve (AUC) analysis. As shown in Figure $2 \mathrm{~A}$, the AUC of serum S100A8 for the prediction of CAP was 0.855 (95\% Cl: 0.791-0.919). A numerical threshold set at $86.89 \mathrm{pg} / \mathrm{ml}$ to minimise the risk of false-negative diagnosis allowed the identification of CAP with $74 \%$ sensitivity and $87 \%$ specificity. Moreover, the AUC of serum S100A8 for the prediction of severity was analysed among CAP patients. As shown in Figure $2 \mathrm{~B}$, the AUCs were as follows: S100A8, $0.893(95 \% \mathrm{Cl}$ : $0.743-$ 0.904); CURB-65, 0.894 (95\% Cl: 0.832-0.954); CRB-65, $0.886 \quad(95 \% \quad \mathrm{Cl}: \quad 0.823-0.950) ; \quad P S I$, 0.939 (95\% Cl: 0.891-0.987); SMART-COP, 0.965 (95\% Cl: 0.932-0.998); CURXO, 0.880 (0.810$0.851)$. In addition, the optimal cut-off value of S100A8 for CAP was $158.32 \mathrm{pg} / \mathrm{ml}$, with a specificity of $78 \%$ and sensitivity of $82 \%$ (Figure 2 B). In order to further rank the predictive capacity of different markers, the machine learning algorithm and bootstrap method were performed. As shown in Figure $2 \mathrm{C}$, we found that the order of rank for different markers were SMART-COP, PSI, CURB-65, S100A8, CRB-65, and CURXO from low to high. Moreover, the predictive capacity of different markers was again tested in a different sample population. The result was similar with the machine learning algorithm (data not shown).

S100A8 knockdown alleviated streptococcus pneumoniae infection-evoked inflammatory cytokines in human pulmonary epithelial cells

To further explore the possible mechanism of S1008A elevation in CAP patients, A549 cells were transfected with $5100 A 8$ siRNA and then exposed to streptococcus pneumoniae to observe whether S100A8 knockdown attenuates streptococcus pneumoniae infection-evoked inflammatory cytokines in human pulmonary epithelial cells. As expected, transfection with S100A8 siRNA obviously decreased S100A8 mRNA in A549 cells (Figure $3 \mathrm{~A}$ ). These results indicated that $5100 \mathrm{~A} 8$ SiRNA transfection significantly and effectively knocked down S100A8 expression in A549 cells. Next, S100A8 and inflammatory cytokines were determined after streptococcus pneumoniae exposure in A549 cells. S100A8 mRNA and supernatant S100A8 levels were all increased after streptococcus pneumoniae exposure in A549 cells (Figures 3 A, B). Interestingly, transfection with S100A8 siRNA obviously alleviated streptococcus pneumoniae-induced upregulation of S100A8 mRNA in A549 cells (Figures $3 \mathrm{~A}, \mathrm{~B}$ ). Moreover, mR- 
A

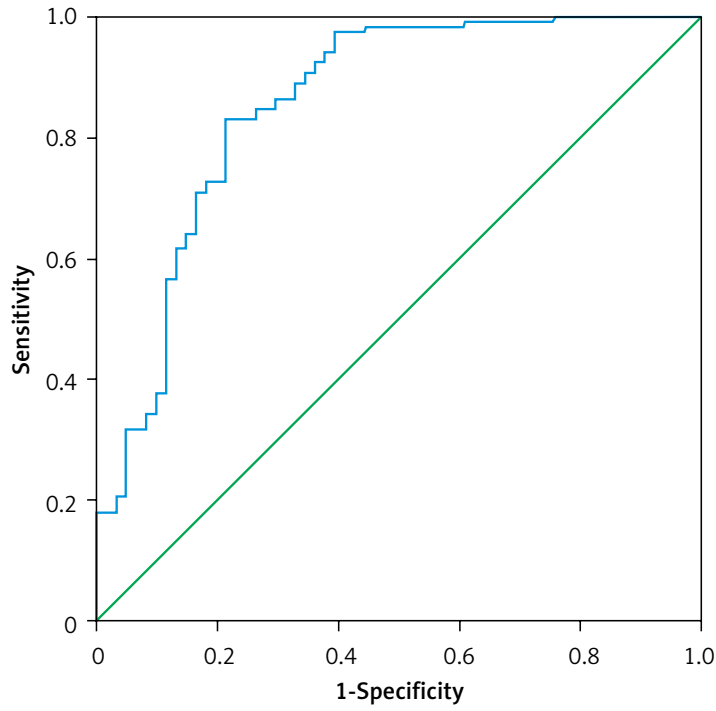

C

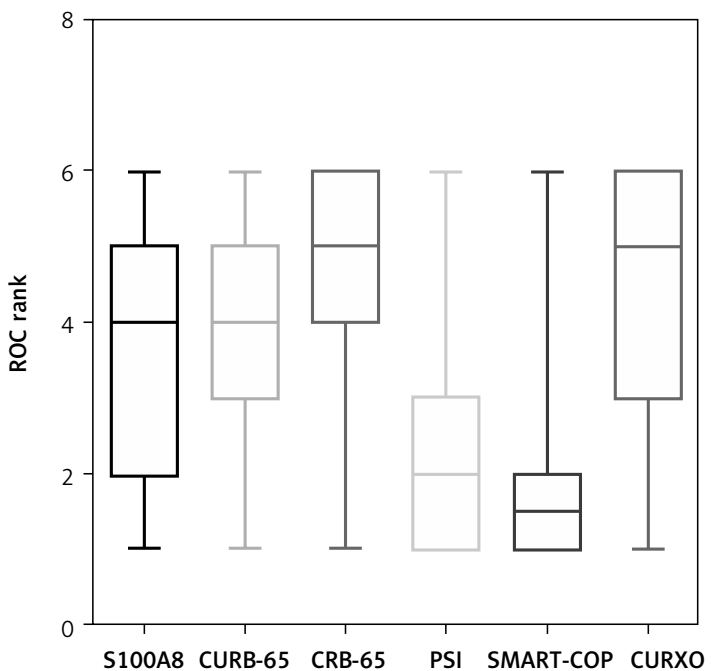

B

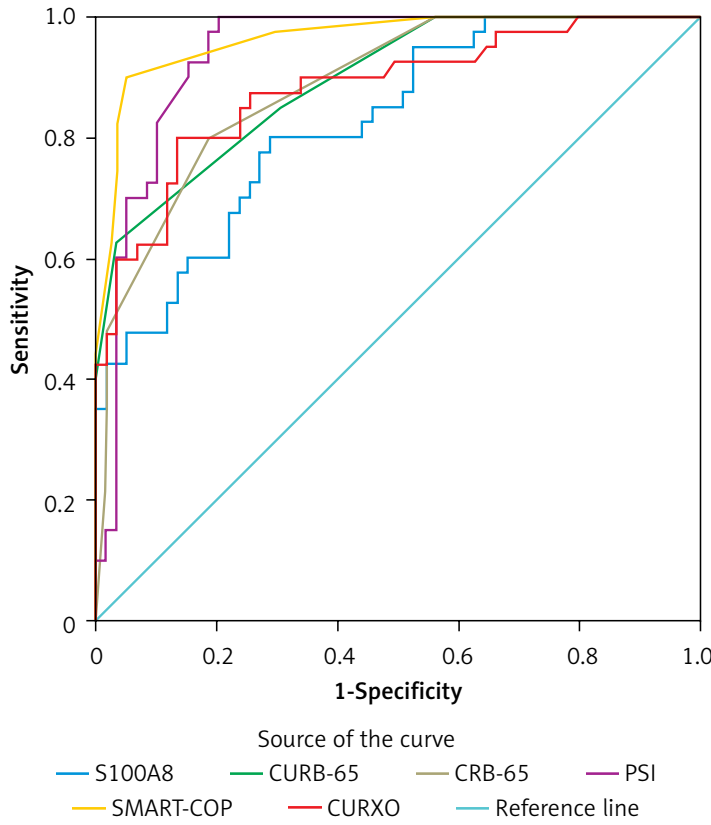

Figure 2. Receiver operating characteristic curves for different predictive biomarkers on admission. A - ROC curve was used to evaluate the diagnostic value of serum S100A8 for CAP. B - ROC curve was used to evaluate the diagnostic value of different predictive biomarkers (S100A8, CRB-65, CURB-65, CURXO, SMART-COP, and PSI) for the severity of CAP. C - The rank of different biomarkers was analysed using the machine learning algorithm and bootstrap method

NAs of inflammatory cytokines (IL-1 $\beta, C R P$, TNF $\alpha$, and $I L-6)$ were elevated in A549 cells after streptococcus pneumoniae infection (Figures $3 \mathrm{C}-\mathrm{F}$ ). Even more impressively, pretreatment with S100A8 siRNA transfection dramatically mitigated streptococcus pneumoniae infection-induced elevation of inflammatory cytokines (Figures $3 \mathrm{C}-\mathrm{F}$ ).

\section{Discussion}

As far as we know, this was the first epidemiological and laboratory study to investigate the association of serum S100A8 and the severity among CAP patients. The major findings of this study include the following: (1) Serum S100A8 heterodimer is increased in CAP patients on admission; (2) Serum S100A8 heterodimer is positively associated with CAP severity scores; and (3) S100A8 knockdown attenuates streptococcus pneumoniae infection-evoked inflammatory cytokines in human lung epithelial cells.

A previous in vivo study found that S100A8 was highly expressed in the pulmonary alveolar walls in mice infected with streptococcus pneumoniae [19]. In vitro experimentation demonstrated that S100A8 protein was increased in the bronchial epithelium after inflammation exposure [20]. In addition, S100A8 was secreted into lung lavage fluid in mice after lipopolysaccharide stimulation [21]. However, there are limited clinical studies exploring the role of S100A8 in CAP patients. In the present research, we found that serum S100A8 heterodimer was increased in CAP patients compared with control subjects. According to the CAP severity indices, serum S100A8 heterodimer gradually increased in parallel with the severity of CAP. Also, there were positive correlations between serum S100A8 heterodimer and the CAP severity scores. Logistical regression analysis further confirmed that serum S100A8 heterodimer was positively associated with CRB-65 score, PSI score, and 
A

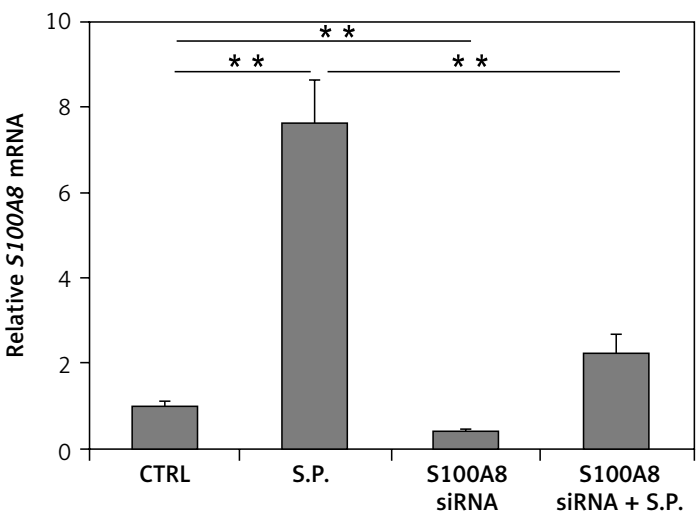

C

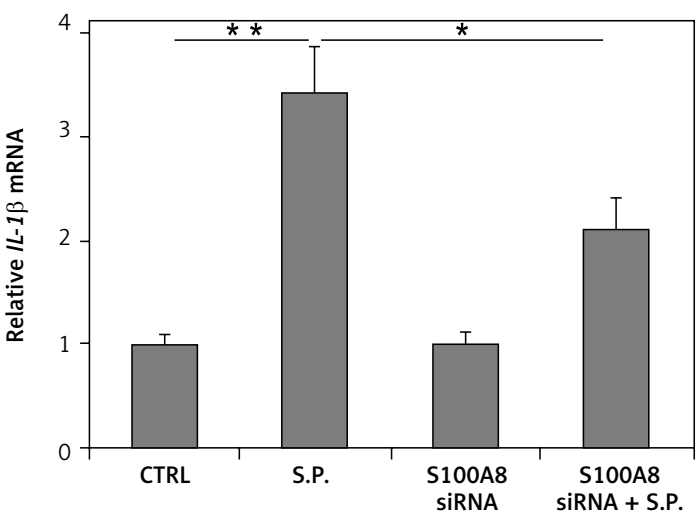

$\mathrm{E}$

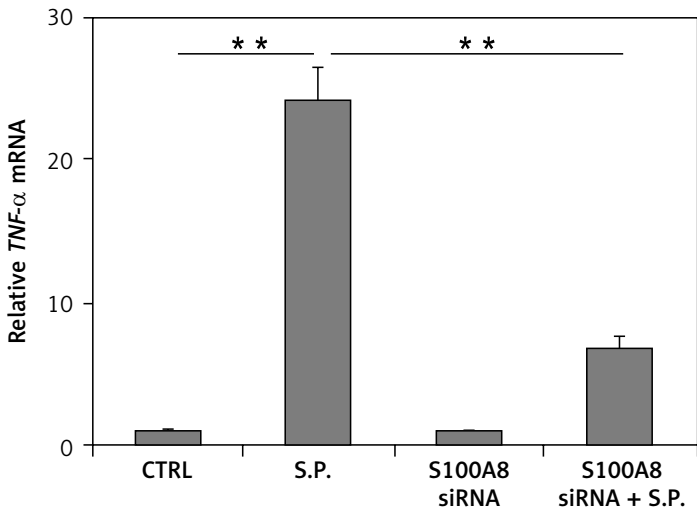

B

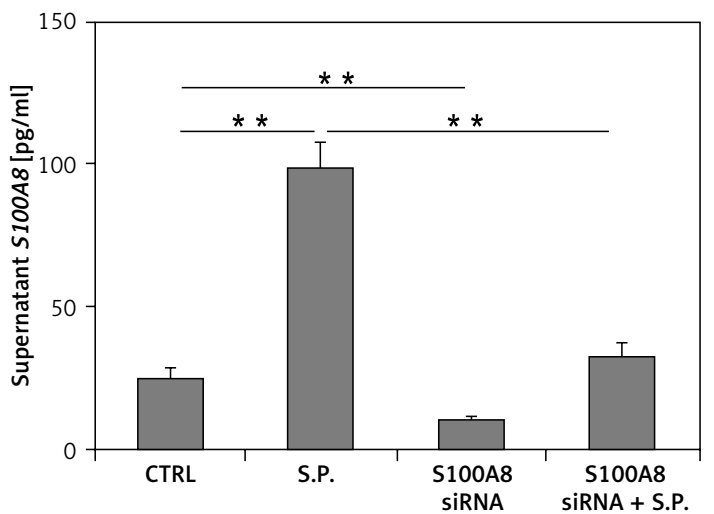

D

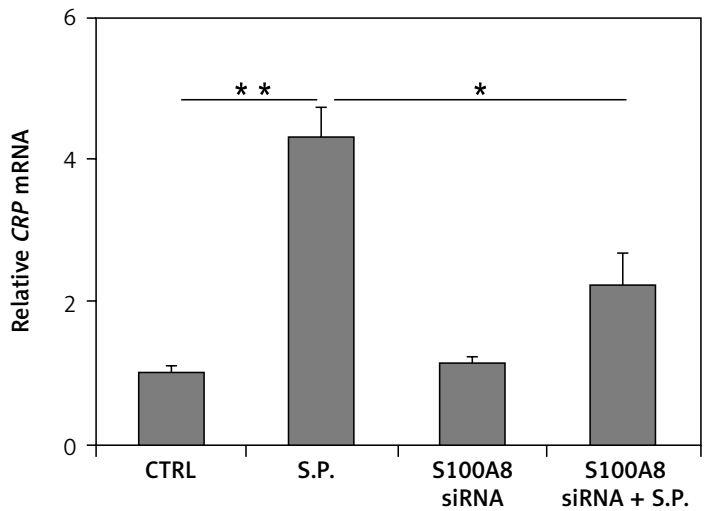

$\mathrm{F}$

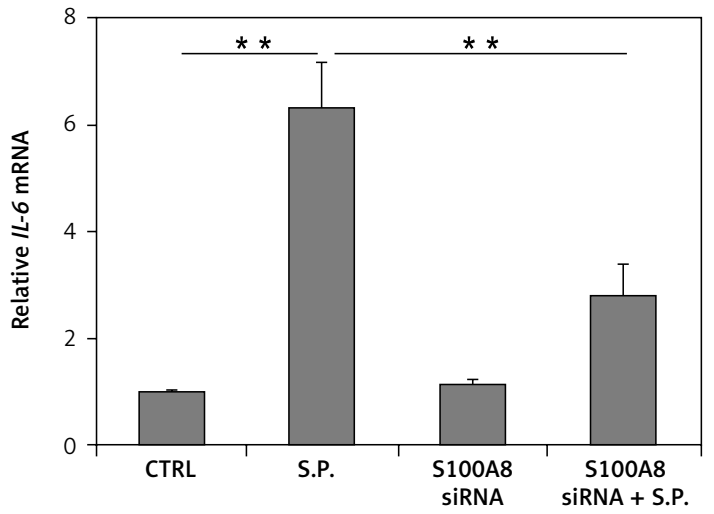

Figure 3. The levels of S100A8 and inflammatory cytokines after streptococcus pneumoniae infection in A549 cells. A - S100A8 mRNA was detected using RT-PCR after streptococcus pneumoniae (S.P.) infection in A549 cells. B - Supernatant S100A8 level was measured using ELISA after S.P. infection in A549 cells. C-F - The mRNAs of inflammatory cytokines were detected using RT-PCR after S.P. infection in A549 cells. C - IL-1 $\beta$. D - CRP. E - TNF- $\alpha$. F - IL-6. All data were represented as mean \pm SEM of six samples $(n=6) .{ }^{*} P<0.05,{ }^{* *} p<0.01$

CURXO score. These results indicate that serum S100A8 heterodimer was positively associated with the severity of CAP.

More and more studies have found that inflammation reaction participates in the pathogenesis of CAP patients. C-reactive protein (CRP) and many pro-inflammatory cytokines (interleukin- $1 \beta$

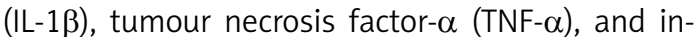
terleukin-6 (IL-6)) were increased in CAP patients [28-30]. Inflammatory cytokines were positively associated with the severity among CAP patients [31]. Serum S100A8 was positively associated with several pro-inflammatory cytokines in inflammatory diseases [32, 33]. However, the associations between serum S100A8 and inflammatory cytokines remained unclear among CAP patients. The present study analysed the associations of serum S100A8 with inflammatory cytokines in CAP patients. These results indicated that serum S100A8 heterodimer was positively associated with TNF- $\alpha, \mathrm{IL}-1 \beta$, and 
CRP. Moreover, a study found that blood routine parameters can be used as indicators for CAP [33]. This study analysed the correlations between serum S100A8 heterodimer and blood routine parameters in CAP patients. We found that there was a weakly positive correlation between serum S100A8 heterodimer with white blood cell, neutrophil-lymphocyte ratio, and monocyte-lymphocyte ratio. These results suggest that serum S100A8 heterodimer could be a diagnostic biomarker for CAP.

A productive relationship between serum S100A8 heterodimer and the CAP severity score indicated that it is a potential predictive biomarker in CAP patients. In the present research, we analysed the predictive power by performing a sensitivity/specificity analysis with ROC curve test. The AUC values always represent the predictive quality. The optimal cut-off value of S100A8 for CAP was 0.225 , with a specificity of $81.6 \%$ and sensitivity of $82.5 \%$. Moreover, we analysed the predictive power for CAP severity S100A8 and the CAP severity score. The results demonstrated that the predictive power was similar between serum S100A8 and CURB-65 score. S100A8 may have better predictive power than CRB-65 and CURXO scores. The machine learning algorithm and bootstrap method further supported our results. Furthermore, we found that the predictive capacity of S100A8 is better than many known biomarkers through a literature review $[33,34]$. Serum S100A8 is more easily detected and obtained than pneumonia severity scores in some cases. Therefore, serum S100A8 heterodimer may have an advantage over CAP severity scores in diagnosing CAP. Consequently, these results imply that serum S100A8 heterodimer can be used as a better diagnostic biomarker in CAP patients.

Despite constant efforts to develop the association of S1008A with CAP and to ascertain the molecular mechanism leading to the pathophysiology of CAP, much remains unknown. Mounting evidence suggests that S100A8 exhibits typical properties of DAMPs, which is released through activating granulocytes, and acts in a cytokine-like manner through binding to cell surface receptors, such as TLR4, CD36, or RAGE. S100A8 can activate several inflammatory signalling pathways and plays critical roles in numerous cellular processes [10-13]. In the inflammatory environment, S100A8 is released and modulates the inflammatory response by stimulating leukocyte recruitment and evoking cytokine secretion. The release of S100A8 can elevate multiple cytokines in inflammatory cells to sustain and exacerbate inflammation $[35,36]$. Earlier research found that S100A8 release induced MyD88 translocation and activated NF- $\mathrm{BB}$ signalling, resulting in TNF- $\alpha$ secretion in phagocytes [37]. Elevated inflammatory host responses result in an unfavourable outcome by driving lung failure and pneumonia. Moreover, our results found that $5100 A 8$ knockdown alleviated streptococcus pneumoniae infection-evoked inflammatory cytokines in human pulmonary epithelial cells. So, we speculate that streptococcus pneumoniae infection induces CAP, partially through S100A8-mediated inflammatory reaction.

In brief, this research found that S100A8 may take part in the development and progression of CAP. These findings may further promote better understanding of the pathogenesis of CAP, and may further the search for potential diagnostic biomarkers for CAP. Nevertheless, there are some flaws in this study. Firstly, this was only a cross-sectional study and in vitro experiment; the causal link between S100A8 heterodimer and CAP patients is needed to demonstrate using further longitudinal studies and animal experiments in the future research. Secondly, this was a single-centre and small-sample study; a larger sample size from multicentre survey is needed in the next work. Thirdly, the mechanism of S100A8-evoked CAP was not clear, more laboratory research is needed in the future. Fourthly, S100A8 heterodimer was only measured in the serum; the levels of S100A8 in sputum and bronchoalveolar lavage fluid are uncharted among CAP patients.

In conclusion, serum S100A8 heterodimer is increased in CAP patients at an early stage. Serum S100A8 heterodimer is positively correlated with the severity of CAP at an early stage. S100A8 knockdown alleviates streptococcus pneumoniaeevoked inflammatory cytokines in the pulmonary epithelial cells, indicating that S100A8 heterodimer may take part in the development and progression of CAP. Consequently, S100A8 heterodimer can be used as an early serum diagnostic biomarker and potentially therapeutic target for CAP in future clinical practice.

\section{Acknowledgments}

Pu Fang and Ling Zheng contributed equally to this work.

This study was supported by National Natural Science Foundation Incubation Program of the Second Affiliated Hospital of Anhui Medical University (grant number: 2019GQFY06).

We greatly appreciate all doctors and nurses of respiratory and critical care medicine in the Respiratory and Critical Care Medicine, the Second Affiliated Hospital of Anhui Medical University for recruiting participators. Moreover, we thank all patients and their families who participated in this research.

\section{Conflict of interest}

The authors declare no conflict of interest. 


\section{References}

1. Esposito S, Cohen R, Domingo JD, et al. Antibiotic therapy for pediatric community-acquired pneumonia: do we know when, what and for how long to treat? Pediatr Infect Dis J 2012; 31: e78-85.

2. Chong CP, Street PR. Pneumonia in the elderly: a review of the epidemiology, pathogenesis, microbiology, and clinical features. South Med J 2008; 101: 1141-79.

3. Wunderink RG, Waterer GW. Community-acquired pneumonia. N Engl J Med 2014; 370: 1861-3.

4. Sahin F, Yıldız P. Distinctive biochemical changes in pulmonary tuberculosis and pneumonia. Arch Med Sci 2013; 9: 656-61.

5. Ewig S, Torres A, Woodhead M. Assessment of pneumonia severity: a European perspective. Eur Respir J 2006; 27: 6-8.

6. Gowin E, Wysocki J, Avonts D, Januszkiewicz-Lewandowska D, Michalak M. Usefulness of inflammatory biomarkers in discriminating between bacterial and aseptic meningitis in hospitalized children from a population with low vaccination coverage. Arch Med Sci 2016; 12: 408-14.

7. Wilson KC, Schünemann HJ. An appraisal of the evidence underlying performance measures for community-acquired pneumonia. Am J Respir Crit Care Med 2011; 183: 1454-62.

8. Stawczyk-Eder K, Eder P, Lykowska-Szuber L, et al. Is faecal calprotectin equally useful in all Crohn's disease locations? A prospective, comparative study. Arch Med Sci 2015; 11: 353-61.

9. Cypers H, Varkas G, Beeckman S, et al. Elevated calprotectin levels reveal bowel inflammation in spondyloarthritis. Ann Rheum Dis 2016; 75: 1357-62.

10. Koy M, Hambruch N, Hussen J, Pfarrer C, Seyfert HM, Schuberth HJ. Recombinant bovine S100A8 and A9 enhance IL-1beta secretion of interferon-gamma primed monocytes. Vet Immunol Immunopathol 2013; 155: 162-70.

11. Loser K, Vogl T, Voskort M, et al. The Toll-like receptor 4 ligands Mrp8 and Mrp14 are crucial in the development of autoreactive CD8+ T cells. Nat Med 2010; 16: 713-7.

12. Wang Y, Fang C, Gao H, et al. Platelet-derived S100 family member myeloid-related protein-14 regulates thrombosis. J Clin Invest 2014; 124: 2160-71.

13. Boyd JH, Kan B, Roberts H, Wang Y, Walley KR. S100A8 and S100A9 mediate endotoxin-induced cardiomyocyte dysfunction via the receptor for advanced glycation end products. Circ Res 2008; 102: 1239-46.

14. Ruan G, Xu J, Wang K, et al. Associations between serum S100A8/S100A9 and knee symptoms, joint structures and cartilage enzymes in patients with knee osteoarthritis. Osteoarthritis Cartilage 2019; 27: 99-105.

15. Nistala K, Varsani H, Wittkowski H, et al. Myeloid related protein induces muscle derived inflammatory mediators in juvenile dermatomyositis. Arthritis Res Ther 2013; 15: R131.

16. Azramezani Kopi T, Amini Kadijani A, Parsian $\mathrm{H}$, et al. The value of mRNA expression of S100A8 and S100A9 as blood-based biomarkers of inflammatory bowel disease. Arab J Gastroenterol 2019; 20: 135-40.

17. Huang $X$, Tan X, Liang Y, et al. Differential DAMP release was observed in the sputum of COPD, asthma and asthma-COPD overlap (ACO) patients. Sci Rep 2019; 9: 19241.

18. Horvath I, Iashchishyn IA, Moskalenko RA, et al. Co-aggregation of pro-inflammatory S100A9 with alpha-synuclein in Parkinson's disease: ex vivo and in vitro studies. J Neuroinflammation 2018; 15: 172
19. Raquil MA, Anceriz N, Rouleau P, Tessier PA. Blockade of antimicrobial proteins S100A8 and S100A9 inhibits phagocyte migration to the alveoli in streptococcal pneumonia. J Immunol 2008; 180: 3366-74.

20. Henke MO, Renner A, Rubin BK, Gyves J, Lorenz E, Koo JS. Up-regulation of S100A8 and S100A9 protein in bronchial epithelial cells by lipopolysaccharide. Exp Lung Res 2006; 32: 331-47.

21. Bozinovski S, Cross M, Vlahos R, et al. S100A8 chemotactic protein is abundantly increased, but only a minor contributor to LPS-induced, steroid resistant neutrophilic lung inflammation in vivo. J Proteome Res 2005; 4: 136-45.

22. Froes F. PSI, CURB-65, SMART-COP or SCAP? And the winner is... SMART DOCTORS. Rev Port Pneumol 2013; 19: $243-4$

23. Maier BB, Hladik A, Lakovits K, et al. Type I interferon promotes alveolar epithelial type II cell survival during pulmonary Streptococcus pneumoniae infection and sterile lung injury in mice. Eur J Immunol 2016; 46: 2175-86.

24. Fu L, Chen YH, Bo QL, et al. Lipopolysaccharide downregulates 11 beta-hydroxysteroid dehydrogenase 2 expression through inhibiting peroxisome proliferator-activated receptor-gamma in placental trophoblasts. J Immunol 2019; 203: 1198-207.

25. Fu L, Chen YH, Xu S, et al. Oral cholecalciferol supplementation alleviates lipopolysaccharide-induced preterm delivery partially through regulating placental steroid hormones and prostaglandins in mice. Int Immunopharmacol 2019; 69: 235-44.

26. Liu H, Liu J, Zhao G. Long non-coding RNA HOTAIR regulates proliferation, migration and invasion of human cervical cancer cells by modulating expression of MAPK1. Arch Med Sci 2019; 16: 1158-65.

27. Fei J, Fu L, Cao W, Hu B, Zhao H, Li JB. Low vitamin D status is associated with epithelial-mesenchymal transition in patients with chronic obstructive pulmonary disease. J Immunol 2019; 203: 1428-35.

28. Loughran AJ, Orihuela CJ, Tuomanen El. Streptococcus pneumoniae: invasion and Inflammation. Microbiol Spectr 2019; 7: 10.1128/microbiolspec.GPP3-0004-2018.

29. Feldman C, Anderson R. Community-acquired pneumonia: pathogenesis of acute cardiac events and potential adjunctive therapies. Chest 2015; 148: 523-32.

30. Torres A, Ramirez P, Montull B, Menéndez R. Biomarkers and community-acquired pneumonia: tailoring management with biological data. Semin Respir Crit Care Med 2012; 33: 266-71.

31. Briel M, Spoorenberg SMC, Snijders D, et al. Corticosteroids in patients hospitalized with community-acquired pneumonia: systematic review and individual patient data metaanalysis. Clin Infect Dis 2018; 66: 346-54.

32. Huang SJ, Ding ZN, Xiang HX, Fu L, Fei J. Association between serum S100A8/S100A9 heterodimer and pulmonary function in patients with acute exacerbation of chronic obstructive pulmonary disease. Lung 2020; 198: 645-52.

33. Życzkowski M, Rajwa P, Burzyński B, et al. Neutrophil-to-mean platelet volume ratio as a new predictor for overall and cancer-specific survival in patients with localized clear cell renal cell carcinoma. Arch Med Sci 2019; 16: 1072-7.

34. Uhel F, Scicluna BP, van Vught LA, et al. Matrix metalloproteinase-8: a useful biomarker to refine the diagnosis of community-acquired pneumonia upon intensive care unit admission? Crit Care 2019; 23: 226. 
35. Lu SM, Yu CJ, Liu YH, et al. S100A8 contributes to postoperative cognitive dysfunction in mice undergoing tibial fracture surgery by activating the TLR4/MyD88 pathway. Brain Behav Immun 2015; 44: 221-34.

36. Shabani F, Farasat A, Mahdavi M, Gheibi N. Calprotectin (S100A8/S100A9): a key protein between inflammation and cancer. Inflamm Res 2018; 67: 801-12.

37. Ulas T, Pirr S, Fehlhaber B, et al. S100-alarmin-induced innate immune programming protects newborn infants from sepsis. Nat Immunol 2017; 18: 622-32. 